\title{
Electrical properties of fiber and carbon nanotube reinforced polymer composites
}

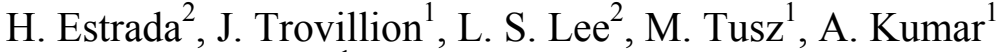 \\ \& L. D. Stephenson ${ }^{1}$ \\ ${ }^{1}$ Construction Engineering Research Laboratory, \\ US Army Engineer Research and Development Center, USA \\ ${ }^{2}$ School of Engineering and Computer Science, \\ University of the Pacific, USA
}

\begin{abstract}
Heightened concerns for electromagnetic interference (EMI) in defense applications have led to developments of multi-functional materials that can provide structural performance while shielding electronic components from electromagnetic waves. Since EMI shielding effectiveness is highly dependent on electrical properties, the goal of this paper is to characterize the conductivity, permittivity, and resistivity of fiber and Carbon Nanotube (CNT) reinforced polymer composites (nanocomposites). The key component of the nanocomposite is a non-woven textile formed using synthetic carbon nanofibers. The material is proprietary and was obtained from Nanocomp Technologies, Inc (www.nanocomptech.com).Electrical testing of specimens was conducted using a low frequency impedance analyser in order to measure the conductivity and resistivity of the nanocomposite for frequencies ranging from $0.1 \mathrm{kHz}$ to $1000 \mathrm{kHz}$. The nanocomposite material's EMI shielding effectiveness is estimated to be approximately $46 \mathrm{~dB}$ for a resistivity of $0.88 \mathrm{Ohms} / \mathrm{square}$. Given the material's average area density of $12.8 \mathrm{~g} / \mathrm{m}^{2}$, these results can be considered promising since the Nanocomp material can be incorporated into conventional composites intended for load bearing applications in order to add EMI shielding to the material with little additional weight.

Keywords: carbon nanotube, composites, electrical properties, electromagnetic interference shielding.
\end{abstract}




\section{Introduction}

The use of carbon nanotubes has great potential in a number of military and civilian applications; particularly when combining CNTs with other materials to develop multifunctional structural materials that integrate multiple attributes synergistically to perform electrical, electromagnetic, thermal, and impact resistant functions, while maintaining minimum strength and stiffness requirements for a given structural application. In this study we concentrate on applications requiring structural performance and shielding from electromagnetic interference (EMI). These two functions can easily be enhanced by embedding CNTs in fiber reinforced polymer materials to produce multifunctional components. Since their discovery by Iijima [6] in 1991, CNTs have been investigated as possible reinforcing materials in polymer based composites [2]. CNTs have been shown to possess many desirable material properties, including high electrical conductivity, high tensile strength, high flexibility, high modulus, and high thermal conductivity. These properties inherent in CNTs allow for significant improvement in mechanical, electrical, and thermal properties of polymers and composites when these materials are loaded with CNTs. However, a number of obstacles exist towards full implementation of CNTs as reinforcing materials. The challenges include a limited understanding of the processes for combining CNTs with composite materials, the need for characterization of the interaction between primary reinforcement (e.g., carbon or glass fibers) and CNTs in a polymer matrix, and significant variations in the properties (physical and chemical) and purity of commercially available CNTs due to their many sources and fabrication techniques; an even larger obstacle to widespread adoption of CNTs is high material cost, which is likely to decrease as volumetric demand increases. Cost constraints aside, once issues related to material quality, as well as interaction, dispersion, and other characteristics have been adequately addressed, CNT additives can be used to enhance electromagnetic shielding of composite materials while improving available strength and stiffness.

\subsection{Electromagnetic interference shielding}

Electromagnetic interference (EMI) is partly caused by rapidly changing voltages and currents in various electrical and electronic devices. Ambient EMI can be considered electromagnetic pollution, most of which consists of spurious, conducted or radiated signals of electrical origin, such as radiation emitted from telecommunications equipment. This EM pollution can adversely alter the operation of sensitive circuits in solid state electronic components found in many defense and civilian instruments. One means of properly protecting this equipment is to utilize materials capable of EMI shielding as containment or housing. In this paper we investigate CNT nanocomposites as potential EMI shielding materials.

There are three mechanisms involved in EMI shielding: reflection, absorption, and multiple reflections of EMI radiation. The reflection mechanism requires materials to possess mobile charge carriers (electrons or holes) to 
interact with incoming electromagnetic radiation waves; that is, electrically conductive materials, which are only required to have a low level of conductivity (or its inverse, resistivity) to provide effective shielding. A volume resistivity of the order of $1.0 \mathrm{ohm}-\mathrm{cm}$ is typically sufficient [4] for effective shielding. The absorption mechanism requires the material to have electric dipoles (materials with high dielectric constant values) and/or magnetic dipoles (materials with high magnetic permeability values) to interact with incoming electromagnetic radiation waves, and is highly dependent on the thickness of the shield. The multiple reflections mechanism requires material to have large surface or interface areas where multiple reflections of radiation waves can occur. In all three mechanisms, shielding is achieved by electromagnetic radiation losses, which are controlled by a material's electrical conductivity and/or magnetic permeability.

Polymers and fibers (glass or carbon) generally contain negligible concentrations of mobile charge carriers or electric dipoles. This lack of mobile charge carriers makes polymer based composites non-conductive (electrically insulating) and thus transparent to electromagnetic radiation. In order for fiber reinforced polymer (FRP) composites to achieve any level of EMI shielding capacity, they must be made electrically conductive by incorporating (loading) intrinsically conductive fillers, such as carbon black, metal particulates, or nickel-coated short carbon fibers [5]. An additional potential polymer filler is CNTs; more specifically, multiwall CNTs (MWCNTs). Previous studies have shown that MWCNT polymer nanocomposites' shielding mechanism is primarily by absorption [7]. Also, the multiple-reflection mechanism's shielding effectiveness is controlled by the shield thickness; if the shield is thicker than the so called "skin depth" the contribution from this mechanism can be ignored [1]. The sum of electromagnetic radiation losses due to reflection, absorption and multiple reflections constitutes EMI shielding effectiveness. These losses are difficult to characterize for CNT nanocomposites since they depend on many factors that are difficult to control; this has been reported in a number of studies where shielding effectiveness values display large variability in similar materials [3]. Some of the more critical factors include processing synthesis, purity of CNTs, and dispersion of CNTs. There are two primary approaches used to predict EMI shielding effectiveness of CNT nanocomposites; one is experimental and one semi-empirical. In both cases, material conductivity plays a key role in EMI shielding effectiveness.

Intuitively, conductivity of polymer based composites should increase as conductive filler loading (filler concentration) is increased. However, full conductivity does not happen instantaneously, a critical filler concentration is needed for the material to display a dramatic increase in conductivity essentially the point at which the material is converted from an insulator to a conductor. This critical concentration is known as the electrical percolation threshold concentration, or the percolation threshold. The percolation threshold is the point at which the filler particles form a continuous 3-D conductive network (percolating networks) throughout the resin matrix. These percolating networks allow electrons to tunnel from one filler particle to another in order to 
overcome the inherent high resistance of the resin matrix. The formation of percolating networks depends primarily on filler's intrinsic conductivity, the particles' geometric aspect-ratio and the distribution of the particles.

Since CNTs have high conductivity and high aspect-ratio, the resulting nanocomposites can be made conductive at a very low percolation threshold provided that the CNTs are uniformly distributed. The conductivity of nanocomposites is also affected by the type of polymer as well as CNT type, surface functionalization, and synthesis method [3]. The polymer type is typically chosen to address a function other than EMI shielding (e.g., structural performance or durability), or it can be selected based on availability and overall cost. CNTs tend to agglomerate because of inherent electrical charge, which adversely affect the uniform dispersion of CNTs throughout the resin materials. One approach used to provide uniform CNT spatial distribution is to chemically functionalize (surface chemical treatment) CNTs. However, this technique disrupts the extended $\pi$-conjugation of CNTs and reduces electrical conductivity. The use of an innovative approach to assemble CNTs into a non-woven textile form to uniformly distribute them in polymer composites is investigated in this study.

CNT based nanocomposites have a number of advantages over conventional metal-based and other EMI shielding materials; including light weight, corrosion resistance, flexibility, and ease of processing. In fact, the use of CNTs can lead to a significant reduction of filler loading required to achieve a desired level of EMI shielding. For instance, percolation thresholds in the range of 5$15 \%$ volume concentration are typical for carbon black filler, and are even higher for dispersed metal particle fillers, 10-30\% [7]. By comparison, $0.3 \%$ weight percolation thresholds for CNTs were found by Trovillion et al. [8], which clearly have a great potential to reduce weight and overall costs if incorporated in FRP composites.

There are two primary approaches used to predict EMI shielding effectiveness of CNT based nanocomposites, one is entirely experimental and one semiempirical. Both are discussed in the next section. The experimental approach is currently underway using a panel that was fabricated using Nanocomp material sandwiched between several glass fiber mats and EMI shielding effectiveness is measured directly. The panel has not been tested to date, but is expected to be tested in the future. In this paper, EMI shielding effectiveness is determined by experimentally measuring conductivity and utilizing a basic theoretical relationship to calculate shielding effectiveness.

\subsection{Characterization of EMI shielding effectiveness}

Direct experimental measurement of EMI shielding effectiveness is based on standard IEEE 299 - Draft standard method for measuring the effectiveness of electromagnetic shielding enclosures, which is specifically intended for enclosures with at least one dimension greater than 2 meters. This standard replaces MIL-STD 285 (1988) and is valid for a frequency range of $9 \mathrm{KHz}$ to $18 \mathrm{GHz}$ and extendable to $50 \mathrm{~Hz}$ and $100 \mathrm{GHz}$, respectively. There is also an ASTM standard that is similar to IEEE 299, ASTM D4935-10 - Standard Test 
Method for Measuring the Electromagnetic Shielding Effectiveness of Planar Materials. EMI work summarized here is based on a procedure from IEEE 299, with a faraday enclosure and aperture that accommodates samples having dimensions of at least 25 inches $(635 \mathrm{~mm})$ by 50 inches $(1270 \mathrm{~mm})$. The primary objective of this test is to determine a panel's contribution to the shielding effectiveness of the faraday cage, assuming ideal behavior, by performing reference and effective measurements, the difference of which is the shielding effectiveness.

Traditionally, EMI shielding effectiveness (or signal attenuation) has been measured using a logarithmic scale, expressed in decibels $(\mathrm{dB})$. This is an effective way to characterize quantities that vary over several orders of magnitude. Using this scale, shielding effectiveness $(S E)$ can be expressed as,

$$
S E=20 \log _{10}\left(\frac{E_{I}}{E_{T}}\right)
$$

where, $E_{I}$ is the incident electric field (reference reading based on open enclosure) and $E_{T}$ is the transmitted electric field (shielded enclosure). Values of $S E$ in the range of $20 \mathrm{~dB}$ to $80 \mathrm{~dB}$ correspond to attenuation of the incident electric field by $90 \%$ to $99.99 \%$; i.e., $S E=20 \mathrm{~dB}$ corresponds to an attenuation of $90 \%, S E=40 \mathrm{~dB}$ corresponds to an attenuation of $99 \%, S E=60 \mathrm{~dB}$ corresponds to an attenuation of $99.9 \%$, and $S E=80 \mathrm{~dB}$ corresponds to an attenuation of $99.99 \%$ of the incident electromagnetic plane wave field. Electromagnetic waves with frequency in the range of $30 \mathrm{MHz}$ to $100 \mathrm{MHz}$ requires $35 \mathrm{~dB}$ to $45 \mathrm{~dB}$ for attenuation; and for critical applications (such as military applications), the attenuation requirements may be as high as $80 \mathrm{~dB}$.

When the electric field measurements have been made using a logarithmic scale, the shielding effectiveness is computed from the reference and shielding measurements (that is, Equation (1) may be written as),

$$
S E=\left|E_{I}\right|(d B)-\left|E_{T}\right|(d B)
$$

where, $\left|E_{I}\right|(d B)$ is the reference measurement, with no panel, in $\mathrm{dB}$ and $\left|E_{T}\right|(d B)$ is the shielding measurement, with panel, in $\mathrm{dB}$. In both cases, the measurements are performed by placing one antenna on the outside of the enclosure (the receiving antenna) and another antenna within the enclosure (the transmitting antenna). A signal is emitted from the transmitting antenna and is measured at the receiving antenna. The difference in performance from a reference (i.e., open aperture) to an experimental state (i.e., closed aperture with the panel being evaluated) is used to calculate the EMI shielding effectiveness. A schematic of the open aperture and closed aperture setup is shown in Figure 1. First, a reference (calibration) measurement is recorded with an open aperture (Figure 1(a)), which establishes a baseline measurement. Then, a measurement is taken again with the shielding panel closing the aperture (Figure 1(b)). 


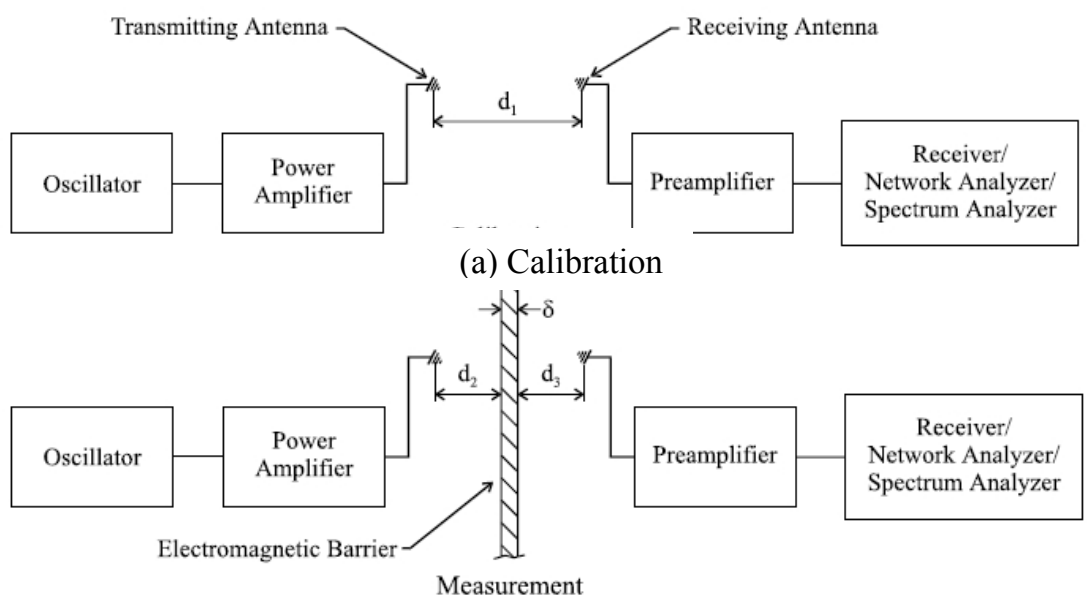

(b) Measurement

Figure 1: Schematic of experimental setup: (a) calibration and (b) measurement.

The panels of interest should be designed for high altitude electromagnetic pulse (HEMP) protection, which are typically required to operate in the range of $0.1-10,000 \mathrm{MHz}$. For this frequency sweep, an antenna configuration that includes low, mid, and high frequencies is required. A list of antennae and corresponding frequencies is shown in Figure 2. The test frequencies, type of measurement, antennas used for measurement, and antenna spacings $\left(\mathrm{d}_{1}, \mathrm{~d}_{2}\right.$, and $\mathrm{d}_{3}$ ) are shown in Figure 1; and are listed in Table 1.

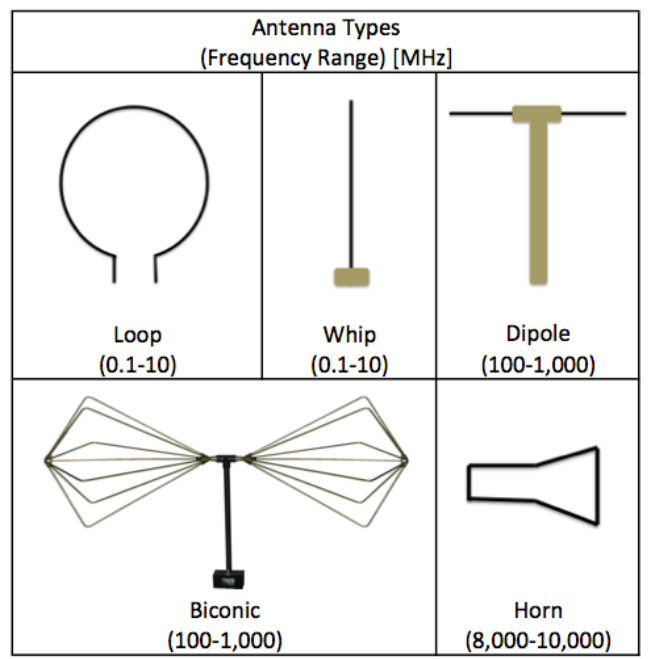

Figure 2: $\quad$ Types of antennae required for panel testing. 
Table 1: $\quad$ Testing rubric for open and closed aperture tests.

\begin{tabular}{|c|c|c|c|c|}
\hline $\begin{array}{c}\text { Frequency } \\
\text { (MHz) }\end{array}$ & $\begin{array}{c}\text { Measurement } \\
\text { Type }\end{array}$ & $\begin{array}{c}\text { Antenna Type } \\
\text { (transmit/receive) }\end{array}$ & $\begin{array}{c}\mathbf{d}_{\mathbf{1}} \\
(\mathbf{m})\end{array}$ & $\begin{array}{c}\mathbf{d}_{\mathbf{2}} \text { and } \\
\mathbf{d}_{\mathbf{3}} \text { (m) }\end{array}$ \\
\hline $\begin{array}{c}0.1 \\
1\end{array}$ & H-field, coplanar & Loop/Loop & 0.6 & 0.3 \\
10 & E-Field, vertical & Whip/Whip & 0.6 & 0.3 \\
\hline $\begin{array}{c}0.1 \\
10\end{array}$ & $\begin{array}{c}\text { Plane wave, } \\
\text { horizontal }\end{array}$ & Biconic/Dipole & 3 & 1 \\
\hline 100 & $\begin{array}{c}\text { Plane wave, } \\
1000\end{array}$ & Horn/Horn & 2.25 & 1.125 \\
\hline 8000 & horizontal & & & \\
\hline 9000 & &
\end{tabular}

In order to properly seal the gap between aperture and panel, a metallic gasket is used. Also, copper tape is applied around the testing panel to ensure contact between the outer edge of the panel and the innermost conductive gasket.

Besides direct experimental measurement, EMI shielding effectiveness can be determined semi-empirically using a measure of a material's conductivity as discussed previously and emphasized in this paper. With the material's resistance, EMI shielding effectiveness can be obtained using the following basic equation [9],

$$
S E=-20 \log \left|\frac{2 R_{A} \eta_{0}}{2 R_{A} \eta_{0}+\eta_{0}^{2}}\right|
$$

where, $R_{A}$ is the sheet resistance in Ohms/square(sheet resistance units) and $\eta_{0}$ is the wave impedance of free space, which is approximately equal to $377 \mathrm{ohms}$. This relationship is used in this study to approximate the EMI shielding effectiveness of the nanocomposite. In fact, using the electrical properties reported by Nanocomp Technologies, Inc (www.nanocomptech.com) for their synthetic carbon nanofibers in a non-woven textile form, average area density of $12.8 \mathrm{~g} / \mathrm{m}^{2}$ and a resistivity of $0.88 \mathrm{Ohms} / \mathrm{square}$, a single sheet could theoretically produce $46 \mathrm{~dB}$ EMI shielding effectiveness. As previously discussed, this corresponds to well over $99 \%$ attenuation of the electromagnetic waves $(S E=40 \mathrm{~dB}$ corresponds to an attenuation of $99 \%)$.

\subsection{Materials}

In addition to the nanocomp sheet, the samples included epoxy resin and fiber glass fabric. To achieve high quality nanocomposite specimens, a low viscosity polymer was used. For this investigation, SC 15 epoxy was selected due to its high strength, low viscosity, and extensive use in structural composite applications. Also, this epoxy system is compatible with the CNT sheet since 
previous studies have shown that CNTs develop good adhesion and wettability with this epoxy. This resin is a low viscosity epoxy resin, specifically developed for the vacuum-assisted resin transfer molding (VARTM) manufacturing processes - a manufacturing process that has been proven cost-effective for applications requiring a low-volume and high-degree of quality control. It is a two phase toughened, amine cured epoxy resin system that exhibits good crosslinking at room temperature. The manufacturer's suggested stoic mixture ratio of resin to hardener is 100:30. This resin is intended for structural and ballistic applications or any application that requires good damage resistance. It allows for a room temperature cure or a post-cure for four hours at $200^{\circ} \mathrm{F}$ to achieve maximum properties; including a modulus of elasticity of $390-\mathrm{ksi}$, elongation of $6.0 \%$, tensile strength of $9.0-\mathrm{ksi}$, and dry glass transition temperature of $180^{\circ} \mathrm{F}$. E-glass fiber reinforcement was obtained from CPIC Fiberglass (www.cpicfiber.com) in woven rovings having a unit weight of $800 \mathrm{~g} / \mathrm{m}^{2}$ and width of $1.27 \mathrm{~m}$. The E-glass woven roving was selected for its excellent wet-out and impregnation characteristics, as well as high mechanical strength and adjustable construction. In general, E-glass fibers have mechanical properties that range from 69-72 $\mathrm{GPa}$ for longitudinal modulus, 3.45-3.9 GPa for tensile strength, and have fiber densities from $2570-2600 \mathrm{~kg} / \mathrm{m}^{3}$.

\section{Sample preparation and testing}

The CNT sheet was used to fabricate nanocomposite panels that were cut into specimens used for electrical testing, which was performed to determine the impedance and resistivity (conductivity) of the material. To produce high-quality specimens the VARTM technique was employed. In order to manufacture the composite panels, the plain-weave E-glass fabric was cut; six layers of the fabric were stacked in alternating warp-fill directions, then the nanocomp film was placed on top and an additional six layers of E-glass fabric were stocked on top. The setup was placed in a vacuum bagging system and infiltrated with SC-15 epoxy. The nanocomposite was then allowed to cure at room temperature for 24 hours, after which the panel was removed from the vacuum bag and post-cured at $100^{\circ} \mathrm{C}$ for one hour.

In this study, two fiber-reinforced panels were fabricated - one panel for electrical testing and one larger (dimensions $1422 \mathrm{~mm}$ by $813 \mathrm{~mm}$ or 56 in by 32 in) panel for EMI shielding effectiveness testing (the test has not been completed to date). A schematic illustration of the VARTM setup is shown in Figure 3.There were three challenges in manufacturing these panels: the static charges during layup caused the nanocomp sheet to crease, it was difficult to develop electrical contact (conductivity) in the panel because the resin is electrically isolating, and the VARTM process had to be modified because the nanocomp is impervious. The nanocomp creasing did not result in loss of electrical conductivity. The second issue was solved by painting the terminals with silver paint after polishing the sample ends using 400-grit sandpaper. For the large panel, this option was impractical and instead, a conductive medium was provided using copper tape along the edges of the nanocomp sheet before it 


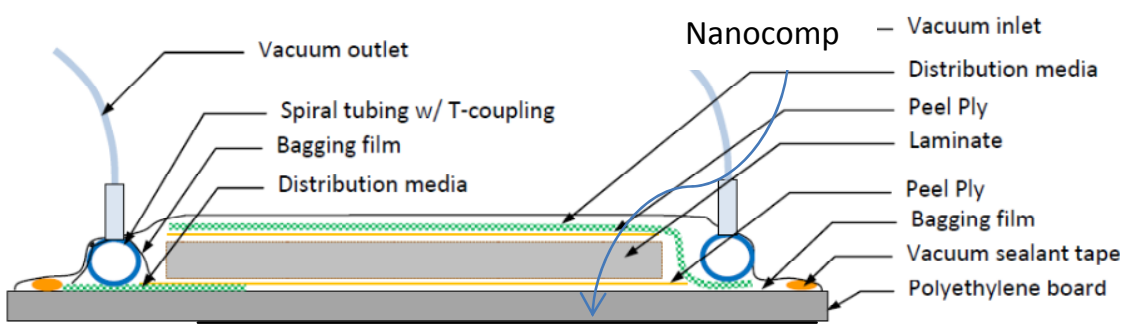

Figure 3: $\quad$ Schematic side view of VARTM fabrication setup.

was embedded in the E-glass layers and the composite impregnated with resin. This copper tape around the edges allows the nanocomp film to come in contact with the external enclosure of the EMI shielding testing equipment and ensures conductivity around the borders of the panel. This will also reduce highfrequency signal leakage through the EMI test aperture. The third issue required the panel to be infused two separate times one for the top six layers and the second time for the bottom six layers.

The CNT nanocomposite electrical properties were determined experimentally using impedance testing of three samples manufactured by VARTM as previously discussed. After curing, the first panel was removed and cut into three specimens of nominal dimensions $20 \mathrm{~mm}$ long, $10 \mathrm{~mm}$ wide, and 1 $\mathrm{mm}$ thick; the actual dimensions were taken at three points and averaged. The ends of each specimen were coated with silver paint to ensure connectivity with the impedance analyzer during measurements (see Figure 4).

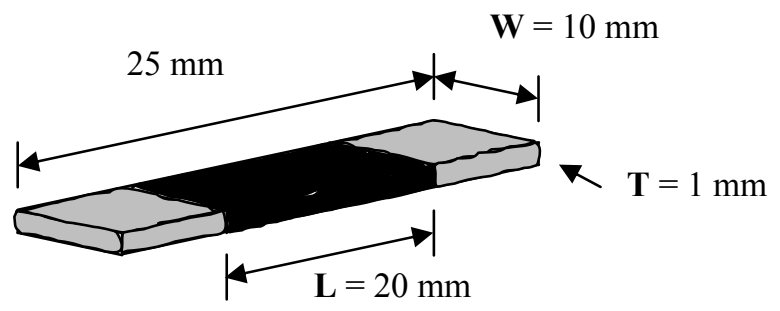

Figure 4: Sample dimensions and preparation procedure for impedance testing.

Electrical testing of specimens was conducted using an HP 4192A low frequency impedance analyzer, which is designed to measure properties such as electrical impedance, phase angle, resistance, conductance, inductance, and capacitance. Impedance (resistance in AC circuits) and resistivity were the only properties investigated since they are considered the primary factors that influence EMI shielding properties. The properties were measured for frequencies ranging from $0.1 \mathrm{kHz}$ to $1000 \mathrm{kHz}$. At low frequencies, the material primarily reflects signals back to the outside, which is done separately for 
electric and magnetic fields. For EMI shielding of magnetic fields, the material needs to have high impedance, while for EMI shielding of electric fields the impedance needs to be low. At higher frequencies, the skin effect is more pronounced in EMI shielding, which means the thickness of the material absorbs the majority of the signal. This consequently requires high conductivity.

\section{Results}

Resistivity (conductivity) and impedance are the most critical electrical properties considered when characterizing EMI shielding effectiveness and the primary parameters investigated in this study. This section summarizes the results of a nanocomp CNT-film nanocomposite. The nanocomp material was used because it is easier to embed CNTs in sheet form than to mix them into the resin. The results for resistivity and impedance for nanocomp fabric laminate samples are shown in Figures 5 and 6, respectively. The figures include the average of a series of three tests and corresponding standard deviation, which is shown as an error bar. The resistivity varied with the orientation of the samples in the impedance analyzer test rig (up to $62 \%$ as shown by the error bar). However, when averaged, the resistivity values converged to results close to those of more repeatable samples.

Comparing these results to other CNT-loaded composite results with $0.8 \mathrm{wt} \%$ non-functionalized MWNT SC-15/SC-780/SC-1 experiments, the nanocomp film exhibits remarkable conductivity, close to that of copper and other highly conductive materials. The results indicate that at all frequencies the average impedance and resistivity are comparable; the low resistivity means that the material is highly conductive. This implies that the nanocomposite is an effective medium for shielding electrical and magnetic fields.

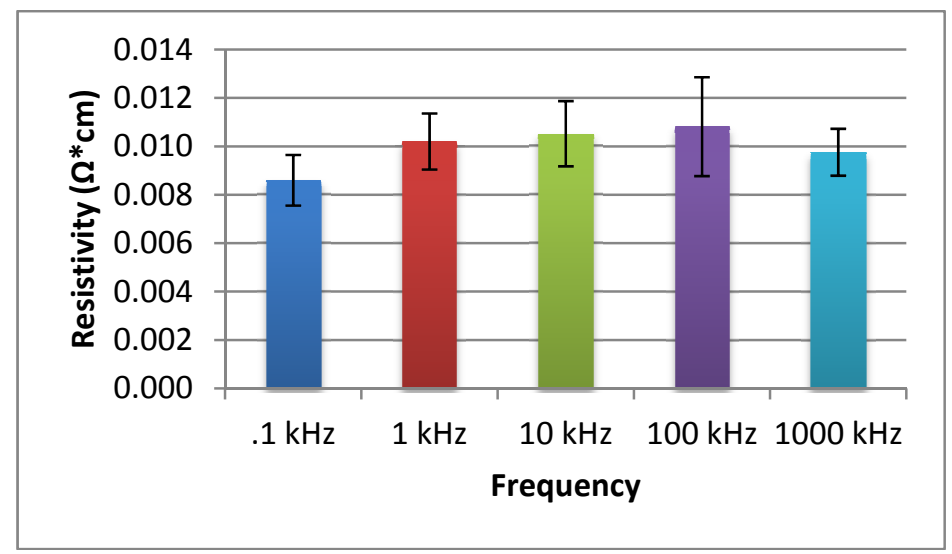

Figure 5: Resistivity of nanocomp composite panel. 


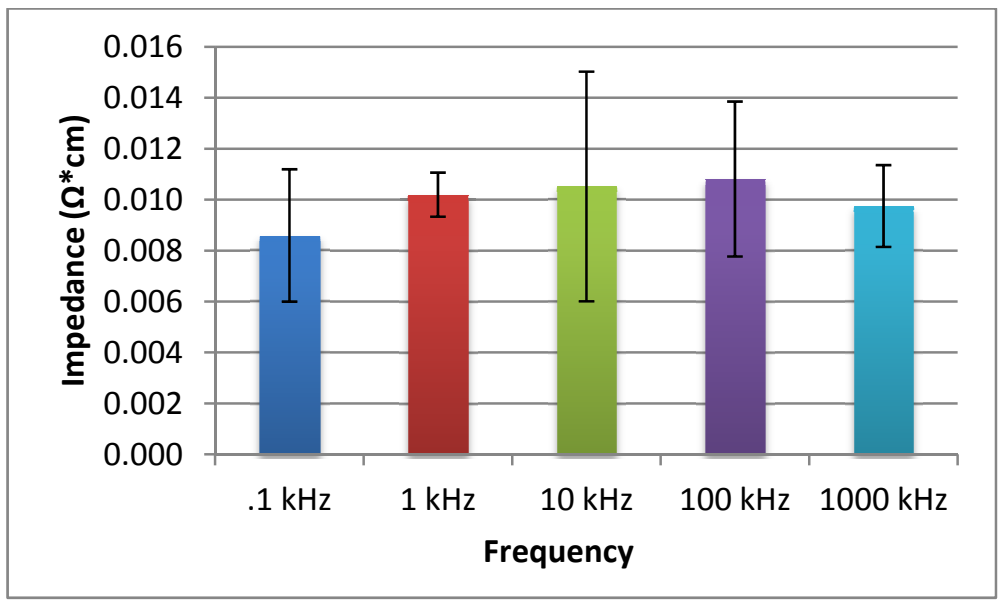

Figure 6: Impedance of nanocomp composite panel.

\section{Conclusions}

This paper presents an overview of the methodology for estimating the EMI shielding effectiveness of nanocomposites. The work emphasizes the experimental measurement of conductivity in order to calculate shielding effectiveness. The paper centers on the development of a material system with electrical properties that can effectively shield EMI waves, which requires a material to be electrically conductive. However, most fiber reinforced polymer composites are not electrically conductive and require that a separate conductive material be integrated within the material. Electrically conductive particulate materials such as CNTs can make epoxy conductive even at small concentrations since electron tunneling occurs even when particles are not in contact. The loading (proportion) of the conductive particulate material required to create electron tunneling and thus make the mixture conductive is the percolation threshold. CNTs only require a small fraction to attain percolation- as little as 0.3 weight percent [8]. However, CNTs must be properly distributed in the epoxy to create tunneling between particles for electrons to travel. This paper presents detailed experimental procedures of the specimen fabrication using nanocomp sheets to integrate the CNTs into the composite. Several conclusions regarding the conductivity of nanocomp based composites can be drawn from the results of this study:

1. One sheet of nanocomp can produce conductivities comparable to that of copper, and can provide up to $99 \%$ EMI shielding.

2. Fabrication of nanocomposites using nanocomp is rather complicated and requires modification of traditional manufacturing techniques, such as VARTM because the nanocomp sheet is impervious to the resin material. 
3. Because the resistivity varied with the orientation of the samples in the impedance analyzer test rig (up to $62 \%$ ), a standardized testing procedure needs to be developed.

4. Lastly, to ensure redundancy with respect to creases and conductivity of the nanocomp sheets in contact with the external enclosure, two layers of nanocomp sheets are recommended.

While these results show that nanocomposites have great promise for use in multifunctional applications, additional work needs to be completed in order to validate the behavior of practical infrastructure components and systems manufactured using these innovative materials. The primary issue is validation of EMI shielding effectiveness using experimental testing.

\section{References}

[1] Al-Saleh, M. H. and Sundararaj, U., Electromagnetic interference shielding mechanisms of CNT/polymer composites. Carbon, 47, pp. 1738-1746, 2009.

[2] Chang, T. E., Kisliuk, A., Rhodes, S. M., Brittain, W. J., and Sokolov, A. P., Conductivity and mechanical properties of well-dispersed single-wall carbon nanotube/polystyrene composite, Polymer, 47, pp. 7740-7746, 2006.

[3] Choudhary, V. and Gupta, A., Polymer/carbon nanotube nanocomposites (Chapter 4). Carbon Nanotube - Polymer Nanocomposites, ed. Yellampalli, S., InTech, intechweb.org, Rijeka, Croatia, 2011.

[4] Chung, D.D.L., Review electromagnetic interference shielding effectiveness of carbon materials. Carbon, 39, pp. 279-285, 2001.

[5] De, S. K. and White, J. R., (eds). Short fiber-polymer composites. Woodhead Publishing Limited: Cambridge, 1996.

[6] Iijima, S., Helical microtubules of graphitic carbon. Nature, 354, pp. 56-58, 1991.

[7] Mamunya, Y., Carbon nanotubes as conductive filler in segregated polymer composites - electrical properties (Chapter 9). Carbon Nanotube - Polymer Nanocomposites, ed. Yellampalli, S., InTech, intechweb.org, Rijeka, Croatia, 2011.

[8] Trovillion J., Estrada, H., Lee, L. S., Tusz, M., Kumar, A., and Stephenson, L. D. Electrical properties of carbon nanotube loaded resins, to be presented at the 2013 SAMPE Annual Conference in Long Beach, CA, May 2013.

[9] Vasquez, H., Espinaza, L., Lozano, K., Foltz, H. and Yang, S., Simple device for electromagnetic interference shielding effectiveness measurement, Proceedings of the IEEE Transactions of Electromagnetic Compatibility; Practical Papers, Articles and Application Notes. 220, pp. 62-68, 2009. 